\title{
Individual differences in visual comparison processes
}

\author{
LYNN A. COOPER \\ University of California, San Diego, La Jolla, California 92093
}

\begin{abstract}
Two experiments are reported in which subjects compared the shape of two successively presented random visual forms. The first stimulus in the pair was one of five "standard" shapes, and the second stimulus was either the same as the standard or different by virtue of a perturbation in shape or an overall reflection. Marked individual differences were found in reaction time for the same-different comparison. For one type of subject "same" responses were faster than "different" responses, speed of "different" responses was unaffected by similarity of the test shape to the standard, and error rates and reaction times were not systematically related. For the other type of subject, "different" responses were generally faster than "same" responses, "different" reaction time decreased as the standard shape and the test shape became increasingly dissimilar, and error rates and reaction times were positively correlated. Implications of these individual differences for models of the same-different comparison process are discussed.
\end{abstract}

A central problem in the area of visual information processing concerns how two visual stimuli are judged to be the same or different. Recently, the nature of the same-different visual comparison process has been studied extensively. Typical variables manipulated include the mode of stimulus presentation (simultaneous and successive) and the degree of difference between the pair of visual stimuli. Most investigators have examined the time required for correct "same" and "different" responses and also error rates on same and different trials. ${ }^{1}$ The results of this research are often conflicting and difficult to summarize briefly. Typically, "same" responses are faster than "different" responses, and "different" reaction times decrease as the stimuli to be compared become less similar. This is true when the stimuli are simple visual forms which vary on a number of well-specified dimensions such as color, shape, ànd size (e.g., Egeth, 1966; Hawkins, 1969; Nickerson, 1967) and also when the stimuli vary on a single dimension such as hue (e.g., Bindra, Donderi, \& Nishisato, 1968). In addition, "same" responses are generally faster than "different" responses when the stimuli consist of strings of letters (e.g., Bamber, 1969) even when the

This research was funded primarily by National Science Foundation Grant GB-31971X to Roger N. Shepard and also in part by National Institute of Mental Health Small Grant MH 25722-01 to the author. James $P$. Cunningham and Roger N. Shepard provided extremely helpful discussions concerning these data and advice concerning interpretation. Peter Podgorny made many exceedingly significant contributions to this research, from assistance in collecting and analyzing the data to fruitful discussion and argumentation concerning interpretations of the results. Requests for reprints should be sent to: Lynn A. Cooper, Department of Psychology, C-009, University of California, San Diego, La Jolla, California 92093. number of unique same and different pairs is equal (Krueger, 1973). The greater speed of "same" responses in visual comparison tasks is not always found, and Bindra, Donderi, and Nishisato (1968) have suggested that easily codable stimuli may produce quicker "same" responses. However, the decrease in "different" reaction time with increasing discriminability between the stimuli to be compared is obtained regardless of stimulus codability (Bindra, Donderi, \& Nishisato, 1968).

Various models of the same-different visual comparison process have been proposed to account for the results described above. (See Nickerson, 1972, for a comprehensive review.) One class of models postulates a single underlying comparison process which determines the speed of both "same" and "different" responses. The underlying process is generally assumed to consist of a self-terminating comparison of the features of the two visual stimuli, either serially (e.g., Egeth, 1966; Nickerson, 1969) or simultaneously (e.g., Hawkins, 1969). These single-process models can predict the relationship between "different" 'reaction time and similarity of a pair of visual stimuli, for as the number of features on which two stimuli differ becomes greater, the earlier a differing feature will be found and the "different" response executed. Unfortunately, this class of models has difficulty predicting the greater speed of the "same" response, for presumably this response occurs only after all features have been compared and no difference has been detected.

A second class of models postulates two independent processes, one underlying the "same" response and the other underlying the "different" response (Bamber, 1969; Bamber \& Paine, 1973; Krueger, 1973; Sekuler \& Abrams, 1968; Tversky, 1969). As 
in single-process models, the "different" processor compares the individual features of the two stimuli and executes the "different" response as soon as a difference is found. The "same" processor operates simultaneously with the "different" processor and gives an output only if the two stimuli are judged to be the same. By assuming independence of the processes underlying the "same" and the "different" responses, these dual-process models can account for all of the major results described above. In addition, dual-process models are consistent with the finding that the speed of "same" and "different" responses can be affected differentially by a given stimulus manipulation (e.g., Egeth \& Blecker, 1971; Hock, 1973; Krueger, 1970, 1973).

The models of the same-different visual comparison process described above are designed to account for the data of an average subject. Implicitly, they assume that the only significant difference between subjects in the same-different comparison task is that of overall speed. Recently, Cooper and Podgorny (in press) have reported qualitative differences between the performance of individual subjects in a visual comparison task which are not easily accommodated by either single-process or dualprocess models.

In the Cooper-Podgorny experiment, six subjects were required to imagine a predesignated random, angular two-dimensional shape rotated from a canonical picture-plane orientation into another designated orientation. When the subject had completed this "mental rotation" (cf. Cooper, 1975; Cooper \& Shepard, 1973; Shepard \& Metzler, 1971), he depressed a response key which provided a measure of the time needed to carry out the mental transformation. Immediately following this first response, a test form was presented in the previously designated orientation and the subject was required to indicate as rapidly as possible, by means of a vocal response, whether the test form was the same as or different from the previously designated random shape. One of five "standard" random shapes, which varied in visual complexity, was used as the first or to-be-transformed stimulus on each trial. For each of the standard shapes, the test probe could be the same as the standard or different by virtue of an overall reflection or a random perturba. tion of the points determining angles of the standard shape. Perturbed test probes varied in their rated similarity to the standard, and six levels of similarity were included. Thus, in the Cooper-Podgorny experiment, the stimuli differed on a continuous dimension of shape. For the purposes of the present paper, only the second, discriminative reaction time will be considered. Results of the Cooper-Podgorny experiment indicated that all of the time required for rotational processing was reflected in the first "preparation" response. Thus, the second "same""different" response provides a measure of the time required to compare a memory representation of a visual form with an externally presented visual test probe and the time required to execute the response.

The discriminative reaction-time data of each of the six individual subjects were analyzed separately, and two distinct patterns emerged. For two of the subjects, "same" responses were faster than "different" responses and "different" reaction time was not affected by the similarity of perturbed test probes to the standard. Cooper and Podgorny (in press) labeled these two individuals "Type I" subjects. For the remaining four subjects, "same" responses were slower than the average "different" response, but faster than "different" responses to highly similar test probes. In addition, for these four subjects, "different" reaction time decreased monotonically with increasing dissimilarity between the perturbed test probes and the standard. These four individuals were labeled "Type II" subjects. The pattern of errors was virtually identical for both types of subjects. Errors io different probes were more frequent than errors 10 same probes, and "different" error rates decreased monotonically as the standard shape and the test probe became more dissimilar. That is, for Type I subjects, reaction times and error rates were uncorrelated, whereas for Type Il subjects there was a positive correlation between reaction times and error rates. For neither type of subject did test-form complexity significantiy affect reaction times or error rates. In addition, overall response speed of Type I subjects was about $200 \mathrm{msec}$ faster than that of Type II subjects.

These differences in performance suggest that Type I and Type II subjects may compare a visual memory representation with an external visual form in qualitatively differene ways. One possibility is that the faster Type I subjects use a holistic process in comparing an internal representation with a visual stimulus, while the slower Type II subjects use a more analytic comparison process. That is, Type I subjects might attempt to match in parallel a memory representation with a visual test form, seeking to verify that the two representations are the same. If this holistic comparison fails to produce a match, then the slower "different" response is made by default. Type II subjects might analytically compare the features of a visual memory representation with a test form, checking for a difference between the two representations. Implications of the Type IType II performance differences for models of same-different visual comparison will be examined in the General Discussion.

The purpose of Experiment I was to explore the generality of the Type I-Type II performance differ- 
Figure 1. The simplest (6-point) standard shape, shown with the seven associated distractors. "D1" through "D6" forms represent random perturbations of the standard varying in similarity to the standard.
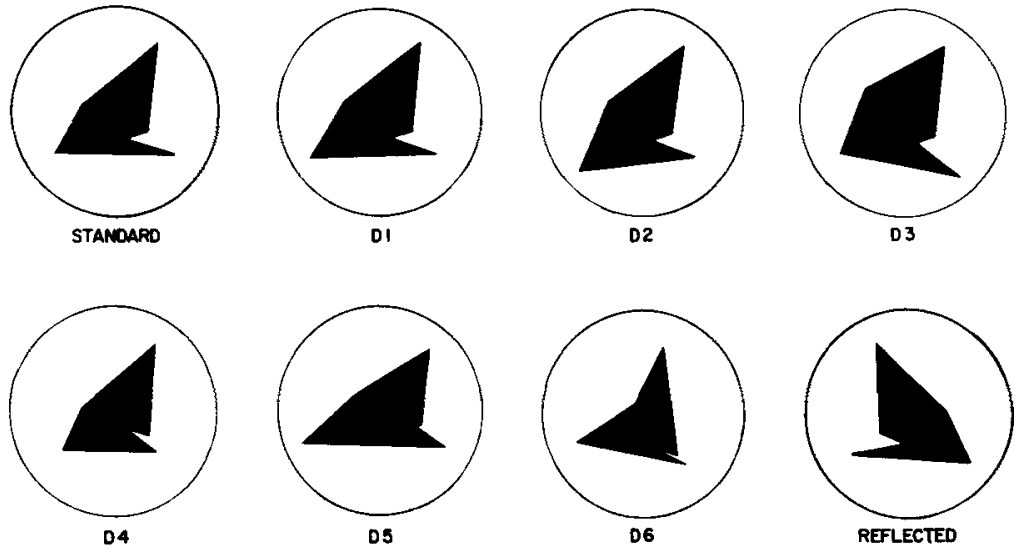

and volunteers from the Stanford area who were paid for their participation in five $1-h$ sessions. Five of the subjects were male, five of the subjects were female, and all of the subjects were right-handed.

Stimuli. The stimulus set was based upon the random, angular shapes used by Cooper (1975) and was identical to the set used by Cooper and Podgorny (in press). Five "standard" shapes were generated by Attneave and Arnoult's (1956) Method I for the construction of random nonsense forms. The shapes varied in the number of angles or points determining inflections on the perimeter, a measure highly correlated with ratings of perceptual complexity of the forms (Antneave, 1957). The five standard shapes in the present experiment were composed of $6,8,12,16$, and 24 angles.

Associated with each of the five standard shapes was a set of seven distractors. One of the distractors was a reflected or mirrorimage version of the standard. The six remaining distractors were random perturbations of the standard shape which varied in their rated similarity to the standard. Figure 1 illustrates the simplest (6-point) standard shape and its associated distractors, and Figure 2 illustrates the most complex (24-point) standard shape and its associated distractors.

The method for generating the perturbations of the standard shapes and the procedture for selecting the six perturbations of each standard included in the stimulus set are described in detail by Cooper and Podgorny (in press). In general, both the number of points perturbed and the extent of each perturbation increases from a "D1" distractor to a "D6" distractor (cf. Figures 1 and 2). In addition, the six perturbations associated with each of the five standard shapes were chosen from a much larger set of perturbations on the basis of ratings of the similarity of each perturbation

\section{Method}

Subjects. The 10 subjects were students at Stanford University

\section{EXPERIMENT I}

24 POINT FOFM
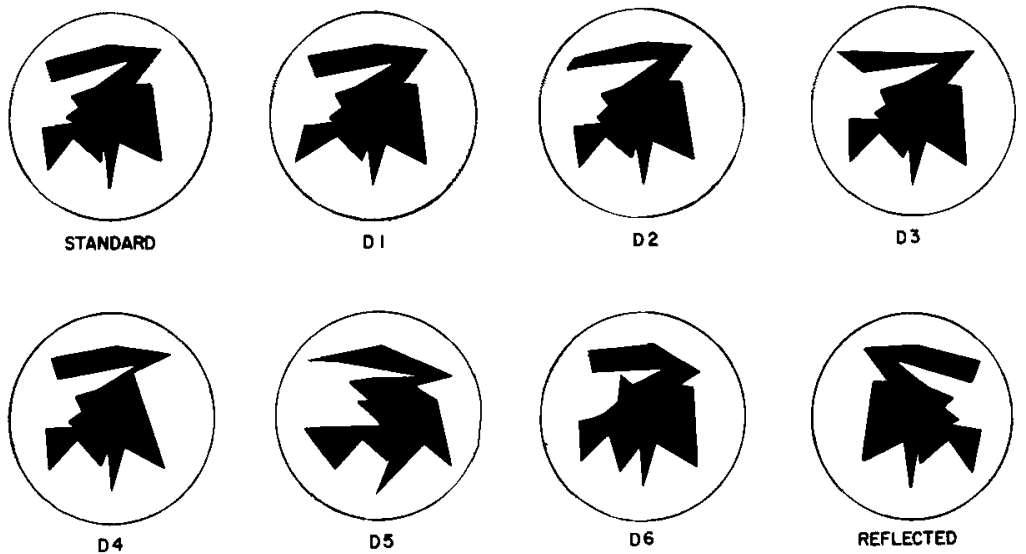

Figure 2. The most complex (24-point) standard shape, shown with the seven associated distractors. "D1" through "D6" forms represent random perturbations of the standard, varying in similarity to the standard. 
to its standard. This selection procedure ensured that rated similarity decreased monotonically and in approximately equal steps from a "D1" to a "D6" perturbation. Thus, the complete set of stimuli consisted of the five standard shapes, their reflected versions, and six perturbations associated with each standard. Some of the perturbations were highly similar to the standards ("D1," "D2") and some of the perturbations were highly dissimilar ("D5," "D6").

The stimuli could be presented at any one of six equally spaced orientations around the circle in the two-dimensional picture plane. These six possible orientations consisted of $60^{\circ}$ steps of clockwise rotation from the arbitrarily chosen $0^{\circ}$ position illustrated in Figures 1 and 2. Six orientations were used for the following reasons: (a) to ensure, as far as possible, that each of the experimental trials was novel, and (b) to replicate, as closely as possible, the stimulus conditions of the Cooper and Podgorny (in press) experiment. The stimuli were presented in an Iconix three-field tachistoscope and appeared centered within an illuminated circular field with a black surround. The circular field subtended a visual angle of $4^{\circ}$, and the stimuli subtended an angle of about $2^{\circ}$. Luminance of the circular field was $68.5 \mathrm{~cd} / \mathrm{m}^{2}$.

Procedure. Each of the 10 subjects was randomly assigned to one of two experimental groups. Group A was composed of three females and two males, and Group B was composed of two females and three males. The structure of each experimental trial was as follows: The subject was informed verbally that the trial was about to begin, and an outline drawing of one of the five standard shapes in one of the six possible picture-plane orientations was presented for a fixed duration of $3,000 \mathrm{msec}$. Immediately following the offset of the standard shape, a blank gray field was presented. The duration of this interstimulus interval was $100 \mathrm{msec}$ for the subjects in Group $\mathrm{A}$ and $3,000 \mathrm{msec}$ for the subjects in Group B. Immediately following the offset of the blank gray field, a test form was presented and the subject determined as rapidly as possible whether the test form was the same as or different from the previously presented standard shape. The test form was always presented in the same orientation as the standard shape, and it could either be the same in shape as the standard or different by virtue of a reflection or a perturbation (cf. Figures 1 and 2).

The subject signaled the "same-different" response vocally, by saying " $S$ " or " $D$," respectively, into a microphone positioned directly below his mouth." This vocal response activated a noiseoperated relay (Hunter sensitivity relay indicator 3203), which triggered the stop on a clock, displaying the total duration of the test form, and terminated the visual display. Thus, reaction time for the same-different discrimination was recorded from the onset of the test form to the subject's response. Verbal feedback concerning the accuracy of the response was given on every trial. Instructions to the subjects emphasized both speed and accuracy of the discriminative response.

Prior to the experimental sessions, subjects were shown illustrations of the stimuli. In addition, each subject participated in 30 practice trials randomly selected from the experimental trials. The composition of the experimental trials was as follows: Each of the five standard shapes was presented 84 times as the first or to-be-remembered stimulus, 14 times in each of the six picture-plane orientations. For any given standard shape presented in any given orientation, on half of the trials the test form was the same as that standard shape and on the other half of the trials the test form was different. Each of the seven types of distractors (six perturbations and one reflection) appeared equally often. Thus, each of the 10 subjects completed 420 experimental trials, as specified by the complete factorial design. The order of trials was randomized anew for each subject. Subjects were informed that the probability of same and different trials was equal.

The experimental trials required five 1 -h sessions for each subject. Each session began with a series of 10 practice trials. Although error reaction 'times were recorded, trials on which errors were made were retaken, randomly interspersed with filler trials, in order to obtain a complete set of error-free data for each subject.

\section{Results and Discussion}

On the basis of the reaction-time criteria outlined above, three of the subjects in the $100-\mathrm{msec}$ interstimulus-interval condition were classified as Type I subjects and two of the subjects were classified as Type II. All five of the subjects in the 3,000-msec-interstimulus-interval condition were classified as Type II. Figure 3 presents the reaction-time data for both types of subjects in the $100-\mathrm{msec}$ condition and for the group data from the $3,000-\mathrm{msec}$ condition. All plotted points are based on correct reaction times only. Although the data are averaged within each type of subject, these average data are quite representative of the data of each of the

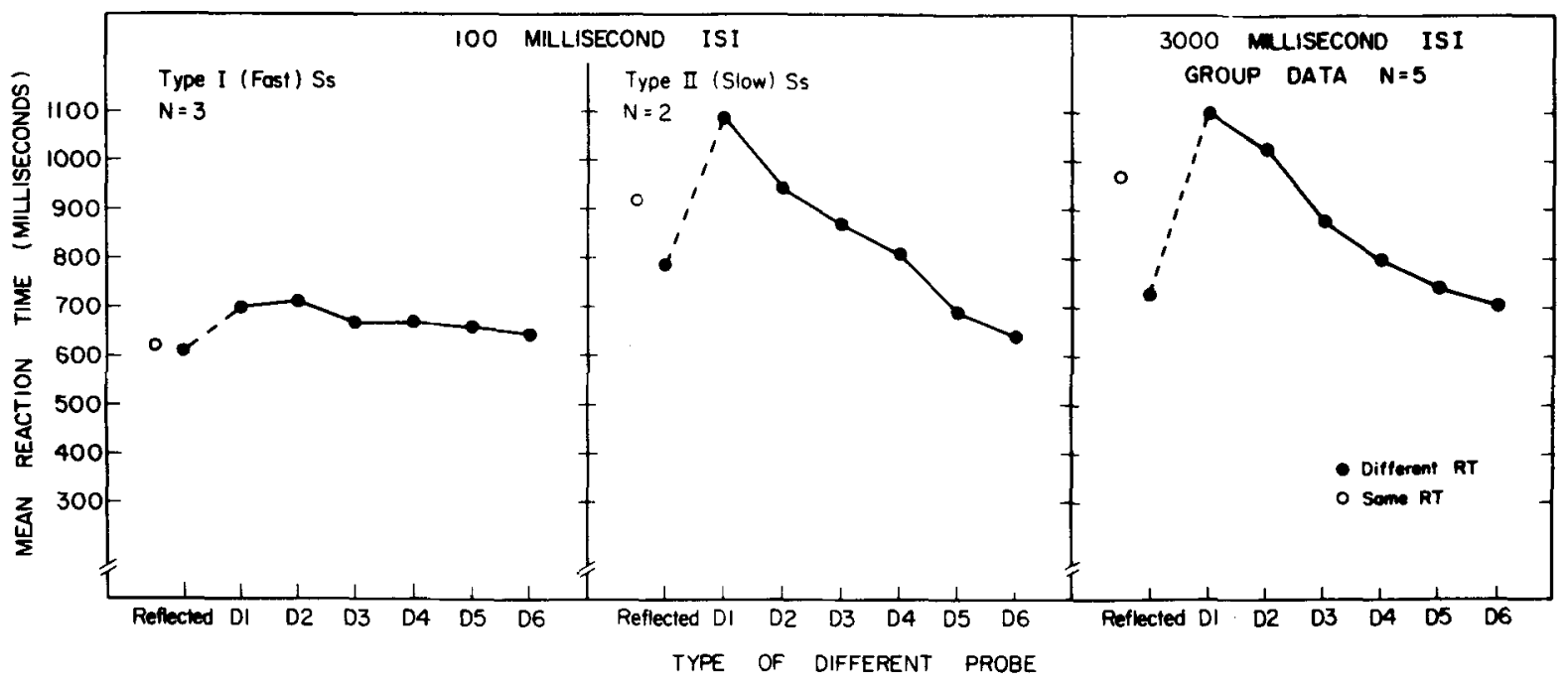

Figure 3. Mean "different" reaction time as a function of type of different probe for Experiment I. Mean "same" reaction times are also shown. Type I subjects in the 100-msec condition are shown in the lefthand panel, Type II subjects in the 100-msec condition are shown in the center panel, and the group data from the 3,000 -msec condition are shown in the right-hand panel. 
Type I and Type II subjects in both interstimulusinterval conditions.

In Figure 3, mean "different" reaction time is plotted as a function of type of different probe-in the left-hand panel for the Type I subjects in the 100 -msec condition, in the center panel for the Type II subjects in the 100 -msec condition, and in the right-hand panel for the group data from the 3,000 -msec condition. The plotted points are averaged over subjects (within each type), test-form complexity, and test-form orientation. Mean "same" reaction times are also shown. Note that for Type I subjects, "same" reaction time is generally faster than "different" reaction times. In addition, overall response speed is faster for Type I subjects than for Type II subjects (cf. Cooper \& Podgorny, in press).

For the Type II subjects in the 100-msec condition and for the 3,000-msec condition group data, "different" reaction time exhibits a sharp monotonic decrease with increasing dissimilarity between the standard shape and the different probe. Average "same" reaction times are of intermediate speed, as are "reflected" reaction times for the Type II subjects in the $100-\mathrm{msec}$ condition. For the group data from the 3,000-msec condition, "reflected" responses are relatively rapid. For Type I subjects, "different" reaction times to all six types of perturbed test probes are approximately equal in speed and "reflected" responses are somewhat faster.

Separate analyses of variance were performed on the "same" and the "different" reaction times of the two types of subjects in the $100-\mathrm{msec}$ condition and on the group data from the 3,000-msec condition. In analyses of "same" responses, the factors were Subjects, Test-Form Complexity, and TestForm Orientation. In analyses of "different" responses, there was an additional factor of Type of Different Probe. Subjects was treated as a random factor in all analyses, and only correct reaction times were included. Consider, first, analyses of the data from the $100-\mathrm{msec}$ condition. For the "same" responses of Type I subjects, the only significant main effect or interaction was the factor of Subjects $[F(2,360)=87.97, p<.01]$. This was true also for the "same" responses of Type II subjects $[F(1,180)=$ $48.61, p<.01]$. For the "different" responses of Type I subjects, the main effect of Subjects was again significant $[F(2,240)=73.72, p<.01]$, as were two two-way interactions with this factor [for Subjects by Test-Form Complexity, $F(8,240)=3.39$, $p<.01$, and for Subjects by Test-Form Orientation, $F(10,240)=2.31, p<.05]$. No other main effects or interactions were significant. For the "different" responses of Type II subjects, two main effects were significant-Subjects and Type of Different Probe [for Subjects, $F(1,120)=10.24, p<.01$, and for Type of Different Probe, $F(6,6)=9.09, p<.01]$. Only one interaction, between the factors of TestForm Complexity and Type of Different Probe, was marginally significant $[F(24,24)=2.08, p<.05]$.

Results of the analyses of the 3,000 -msec condition were similar to those of the Type II subjects in the 100-msec condition. For "same" responses, the only significant main effects were Subjects and Test-Form Complexity $[F(4,720)=134.34, p<.01$, and $F(4,16)=7.85, p<.01$, respectively], and the only significant interaction was between these same two factors $[F(16,720)=6.07, p<.01]$. For "different" responses, only the main effects of Subjects and Type of Different Probe achieved statistical significance $[F(4,480)=110.08, p<.01$, and $F(6,24)=$ $16.23, p<.01$, respectively]. The only significant interactions were between Test-Form Complexity and Type of Different Probe $[F(24,96)=3.09$, $p<.01]$ and the three-way interaction of these two factors with the factor of Subjects $[F(96,480)=1.56$, $p<.01]$.

Table 1 presents error rates to same test forms, different test forms, and the seven types of different probes separately for the Type I and Type II subjects in the 100-msec condition and for the group data from the 3,000-msec condition. For the $100-\mathrm{msec}$ condition, Type 1 subjects are generally more accurate than Type Il subjects. However, despite the marked differences in the reaction-time data for these two types of subjects (cf. Figure 3), the patterns in their error rates are quite similar. Both types of subjects make more errors to different than to same test forms, and for both types of subjects error rates decrease monotonically with increasing dissimilarity between the standard shape and the test probe. For subjects in the 3,000 -msec condition, error rates to

Table 1

Error Rates to Same Test Forms, Different Test Forms, and the Seven Types of Different Probes for Type I Subjects in the 100 -msec Condition, Type II Subjects in the 100-msec Condition and the Group Data for the 3,000-msec Condition (Experiment I)

\begin{tabular}{lccc}
\hline & \multicolumn{2}{c}{$100-\mathrm{msec}$ ISI } & \\
\cline { 2 - 3 } & $\begin{array}{c}\text { Type I } \\
\text { Subjects } \\
(\mathrm{n}=3)\end{array}$ & $\begin{array}{c}\text { Type II } \\
\text { Subjects } \\
(\mathrm{n}=2)\end{array}$ & $\begin{array}{c}\text { 3,000-msec ISI } \\
\text { Group Data } \\
(\mathrm{n}=5)\end{array}$ \\
\hline Same & 7.08 & 11.39 & 9.09 \\
Different & 8.56 & 15.15 & 8.79 \\
D1 & 26.23 & 33.33 & 31.19 \\
D2 & 14.27 & 27.71 & 14.28 \\
D3 & 6.25 & 10.45 & 5.66 \\
D4 & 4.25 & 7.69 & 1.96 \\
D5 & 1.10 & 3.22 & \\
D6 & & & \\
Reflected & 1.10 & 9.09 & .66 \\
\hline
\end{tabular}

Note-Numbers are expressed in percentages. 
same and to different test forms are virtually equal. In addition, error rates to different test forms decrease as the standard shape and the test form become less similar.

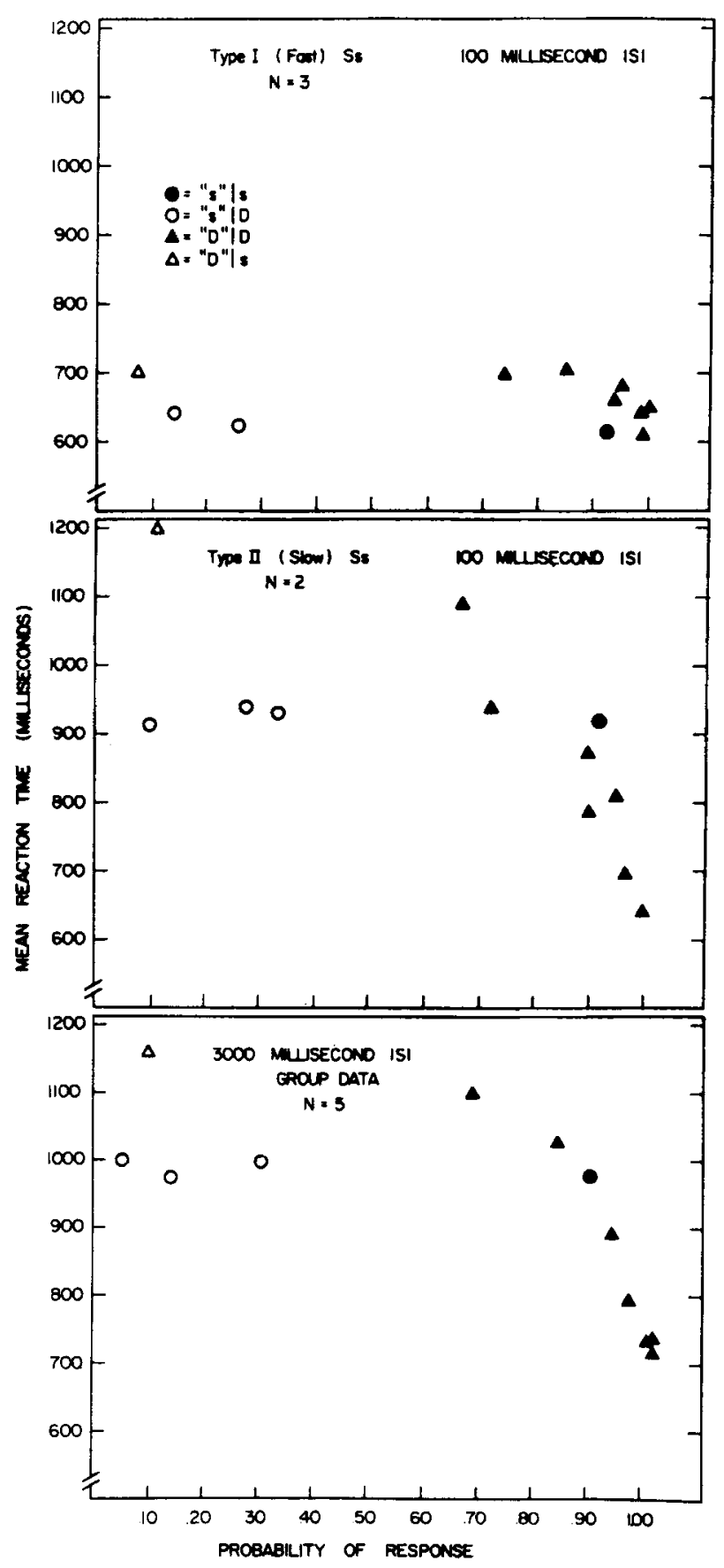

Figure 4. Mean reaction time as a function of probability of the response. Both correct and incorrect reaction times are plotted. Type I subjects in the 100 -msec condition are shown in the upper panel. Type II subjects in the $100-\mathrm{msec}$ condition are shown in the center panel, and the group data from the 3,000 -msec condition are shown in the lower panel.
In Figure 4, mean reaction time is illustrated as a function of response probability separately for the Type I and Type II subjects in the $100-\mathrm{msec}$ condition and for the group data from the 3,000-msec condition. Note that both correct ("same" I same and "different" | different) and incorrect ("same" | different and "different"' | same) reaction times are plotted. Filled symbols represent correct responses, and unfilled symbols represent incorrect responses. Mean reaction time for correct "same" responses is plotted, and mean reaction times for correct "different" responses are plotted separately for each of the seven types of different probes. Similarly, mean reaction time for incorrect "different" responses is plotted, and mean reaction times for incorrect "same" responses are plotted separately for the various types of different probes. Only data points with five or more observations are included, so the incorrect "same" responses which are plotted represent error reaction times to different probes which are highly similar to the standard shape.

For the Type I subjects, both correct and incorrect reaction times fall within a 100 -msec range, and "same" responses (both correct and incorrect) are generally faster than "different" responses (both correct and incorrect). For the Type II subjects in the 100-msec condition and for the group data from the 3,000-msec condition, the speed of the "different" response is strongly related to the probability of the response. This is a reflection of the facts that (a) correct reaction time to different probes decreases and accuracy increases with increasing dissimilarity between the standard shape and the test form, and (b) incorrect "different" reaction time is the longest response made and occurs with low probability. Note, however, that the speed of the "same" response is not systematically related to the probability of the response. All "same" responses, whether correct or incorrect, are executed with approximately equal speed. Implications of these reaction-time-probability data for the nature of Type I-Type II processing differences will be outlined in the General Discussion.

In summary, the performance differences reported by Cooper and Podgorny (in press) were obtained in the present experiment only when the interval between presentation of a standard shape and presentation of a test probe was short. When the interstimulus interval was extended to $3,000 \mathrm{msec}$, all subjects exhibited Type II performance. At first blush, these results seem to suggest that the holistic sort of processing hypothesized to underlie Type I performance is possible only when a test form is compared with a memory representation of an immediately preceding visual shape. Alternatively, the above results might simply be explained by a sampling problem. That is, it may be that the length 
Figure 5. Mean "different" reaction time as a function of type of different probe for Experiment II. Mean "same" responses are also shown. In the left-hand panel, the data for Type I subjects are shown for both interstimulus intervals. In the right-hand panel, the data for Type II subjects are shown for both interstimulus intervals.

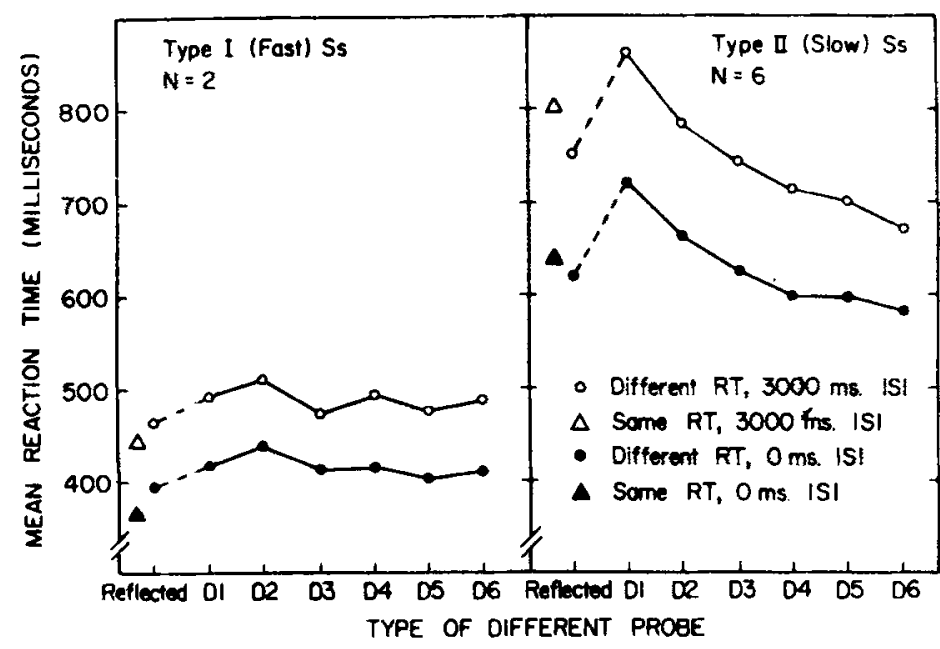

of the memory retention interval has no effect on the way in which a visual test form is compared with a memory representation. Rather, all of the five subjects in the 3,000-msec condition may have been of the Type II variety and, thus, would have exhibited the same performance with a short memory interval. In order to evaluate this possibility, a second experiment was conducted with a new group of subjects, and length of the interstimulus interval was varied within subjects.

\section{EXPERIMENT II}

\section{Method}

Subjects. The eight subjects were students and staff at Stanford University and volunteers from the Slanford area who were paid for their participation. Five of the subjects were female, and three were male. All of the subjects were right-handed. None of the subjects had participated in Experiment I.

Stimuli. The stimuli and the apparatus were identical to those used in Experiment $\mathrm{J}$. In order to reduce the number of trials per subject, standard shapes and test forms were presented in one of three equally spaced picture-plane orientations. The three possible orientations consisted of $120^{\circ}$ steps of clockwise rotation from the arbitrarily chosen $0^{\circ}$ position illustrated in Figures 1 and 2.

Procedure. The structure of the experimental trials, the nature of the required response, the composition of practice trials and the instructions to the subjects were identical to those of Experiment 1 . For each of the eight subjects, two interstimulus intervals -0 and $3,000 \mathrm{msec}-$ were used. Within each of the interstimulus-interval values, each of the standard shapes was presented 42 times as the first or to-be-remembered stimulus, 14 times in each of the three picture-plane orientations. For any given standard shape presented in any given orientation, on half of the trials the test form was the same as that standard shape and on the other half of the trials the test form was different. Each of the seven types of different probes appeared equally often. Thus, each of the eight subjects completed 420 experimental trials, as specified by the complete factorial design.

The experimental trials required five $I$-h sessions for each subject. Trials were blocked by condition $(0-\mathrm{msec}$ interstimulus interval and 3,000-msec interstimulus interval), and two blocks of each type of trials were run in each session. Order of blocks was balanced across sessions, and the order of trials within each block was random. As in Experiment $I$, error reaction times were recorded and error trials were retaken.

\section{Results and Discussion}

Two of the eight subjects were classified as Type I, and six were classified as Type II. Each of the subjects showed the same pattern of performance in both the 0 - and the 3,000-msec-interstimulus-interval conditions. Figure 5 presents the correct reactiontime data for both types of subjects and for both interstimulus intervals. Although the data are averaged over subjects within each group, the average data for each type of subject are representative of the data of each individual subject in the group.

In Figure 5, mean "different" reaction timeaveraged over subjects, test-form complexity, and test-form orientation-is plotted as a function of type of different probe. Mean "same" reaction times are also shown. For the Type I subjects shown in the left-hand panel, "same" reaction times are faster than "different" reaction times. For the Type Il subjects shown in the right-hand panel, "same" reaction times are of intermediate speed. For both types of subjects, the speed of both "same" and "different" responses is slower with the longer interstimulus interval. In addition, overall response speed is faster for Type I than for Type II subjects for both interstimulus intervals (cf. Figure 3; Cooper \& Podgorny, in pess). As in Experiment 1, "different" reaction times of the Type I subjects are not systematically related to type of different probe. For the Type II subjects, "different" reaction time decreases markedly with increasing dissimilarity between the standard shape and the test form.

Separate analyses of variance were performed on the "same" and the "different" reaction times for the two types of subjects. In analyses of "same" responses, the factors were Subjects, InterstimulusInterval Conditions, Test-Form Complexity, and 
Table 2

Error Rates to Same Test Forms, Different Test Forms, and the Seven Types of Different Probes for Type I Subjects and Type II Subjects for 0-msec and 3,000-msec Conditions (Experiment II)

\begin{tabular}{lrrrr}
\hline & \multicolumn{2}{c}{ 0-msec ISI } & & \multicolumn{2}{c}{$3,000-\mathrm{msec}$ ISI } \\
\cline { 2 - 3 } \cline { 5 - 6 } & $\begin{array}{c}\text { Type I } \\
\text { Subjects } \\
(\mathrm{n}=2)\end{array}$ & $\begin{array}{c}\text { Type II } \\
\text { Subjects } \\
(\mathrm{n}=6)\end{array}$ & $\begin{array}{c}\text { Type I } \\
\text { Subjects } \\
(\mathrm{n}=2)\end{array}$ & $\begin{array}{c}\text { Type II } \\
\text { Subjects } \\
(\mathrm{n}=6)\end{array}$ \\
\hline Same & 10.64 & 8.43 & 10.09 & 11.52 \\
Different & 9.09 & 6.53 & 13.39 & 11.02 \\
D1 & 23.08 & 22.71 & 39.31 & 29.67 \\
D2 & 14.29 & 10.87 & 23.05 & 17.43 \\
D3 & 11.76 & 2.17 & 11.76 & 8.16 \\
D4 & 6.25 & 2.17 & 8.92 & 7.22 \\
D5 & 3.23 & & 3.23 & \\
D6 & & 1.10 & & \\
Reflected & & 2.17 & 3.23 & 5.26 \\
\hline
\end{tabular}

Note-Numbers are expressed in percentages.

Test-Form Orientation. In analyses of "different" responses, there was an additional factor of Type of Different Probe. The factor of Subjects, was treated as a random effect in all analyses, and analyses of variance were performed on correct reaction times only. For the "same" responses of Type I subjects, only the main effects of Subjects and Conditions $(0-$ and $3,000-\mathrm{msec}$ interstimulus intervals) were significant $[F(1,180)=4.17, p<.05$, and $F(1,1)=$ $167.89, p<.05$, respectively]. No interactions achieved statistical significance. For the "same" responses of Type II subjects, these same two main effects were significant [for Subjects, $F(5,900)=$ $91.95, p<.01$, and for Conditions, $F(1,5)=20.21$, $p<.05]$. No other main effects were significant, and only one interaction-Subjects by Conditions-was significant $[F(5,900)=13.04, p<.01]$. For the "different" responses of Type I subjects, the only main effect or interaction to achieve significance was the factor of Subjects $[F(1,48)=10.82, p<.01]$. For the "different" responses of Type II subjects, three main effects-Subjects, Test-Form Complexity, and Type of Different Probe-were significant $[F(5,240)=108.94, \quad p<.01, F(4,20)=5.06$, $p<.01$, and $F(6,30)=6.94, p<.01$, respectively $]$. There was one significant interaction between the factors of Test-Form Complexity and Type of Different Probe $[F(24,120)=2.57, p<.01]$.

In Table 2, error rates are presented for Type I and Type II subjects for both interstimulus intervals. As in Experiment I, the patterns of errors are quite similar for the two types of subjects despite the considerable differences in their reaction-time data. For both types of subjects and for both interstimulus intervals, error rates to different test probes decrease as the standard shape and the test probe become less similar. Unlike Experiment I, both types of subjects make slightly more errors to same than to different test forms, with the exception of Type I subjects in the 3,000-msec condition. In addition, Type II subjects are slightly more accurate than Type I subjects. For both types of subjects, error rates are higher with the longer interstimulus interval.

In Figure 6, mean reaction time is plotted as a function of probability of response separately for the Type I and Type II subjects and for both interstimulus intervals. These plots correspond to those in Figure 4 from Experiment I, and both correct and incorrect reaction times are shown. Only data points with five or more observations are plotted, so the incorrect "same" responses represent error reaction times to different probes which are highly similar to the standard shape. As in Experiment I (cf. Figure 4), both correct and incorrect responses of Type I subjects for both interstimulus intervals fall within a very small range. For Type II subjects, both correct and incorrect "same" responses are of approximately equal speed. The speed of "different" responses varies considerably. Correct "different" reaction times are related to response probabilities which, in turn, are related to the similarity between the standard shape and the test form. For Type II subjects under both interstimulus-interval conditions, the longest response to be made is the incorrect "different"' response.

In summary, the Type I-Type II performance difference has emerged clearly from the data of Experiment II. In addition, these data discredit the notion that length of retention interval affects the nature of comparison strategies. Length of retention interval did have an effect on mean reaction time, but that effect was confined to an overall decrease in reaction time, for both types of subjects, with the short retention interval. The qualitative differences in the performance of Type I and Type II subjects persisted at the 3,000 -msec interstimulus interval.

\section{GENERAL DISCUSSION}

The marked individual differences in performance on same-different visual comparison reported by Cooper and Podgorny (in press) have been replicated in the two experiments reported here. Contrary to prior expectations, these differences were apparent under conditions of both long and short intervals between presentation of the standard shape and presentation of the test probe. The central features of these performance differences, illustrated in Figures 3 through 6 and in Tables 1 and 2, can be summarized as follows. For Type I subjects: (a) correct "same" reaction time is faster than correct "different" reaction time; (b) correct "different" reaction time is not affected by similarity of the test probe to the standard shape; (c) incorrect "same" and "diferent" reaction times are approximately equal to 
correct reaction times; (d) error rates and reaction times are not systematically related; and (e) overall response speed is quite fast. For Type II subjects: (a) correct "same" reaction time is slower than average correct "different" reaction time, but faster than "different" reaction time to test probes which are highly similar to the standard shape; (b) correct "different" reaction time decreases monotonically as the standard shape and the test probe become increasingly dissimilar; (c) incorrect "same" reaction times are approximately equal to correct "same" reaction time, but incorrect "different" reaction time is longer than all correct "different" reaction times; (d) error rates and reaction times are positively correlated; and (e) overall response speed is relatively slow.

In addition, analyses of the correct "different" responses of Type II subjects consistently revealed a significant interaction between the factors of TestForm Complexity and Type of Different Probe. Analyses of "different" reaction times of Type I subjects never showed this interaction. If we consider the fact that only for Type II subjects were response times affected by similarity between the test probe and the standard shape, then this interaction can be explained in the following way. Despite the rating procedure used to select the test stimuli, it is still possible that the sizes of the similarity steps from D1 perturbations to D6 perturbations (cf. Figures 1 and 2) were unequal both within a given standard shape and between standards at the five complexity levels. If this were true, then these unequal similarity steps should have affected the "different" reaction times of Type 11 subjects. In order to evaluate this possibility, 10 naive subjects were asked to make a complete rank ordering of the similarity between 30 pairs of shapes-each of the five standards paired with each of its six perturbations. These similarity rankings $(1=$ most similar pair, $30=$ least similar pair) were then correlated with rank order of "different" reaction-time speed for each perturbation at each of the complexity levels $(1=$ slowest response, $30=$ fastest response $)$. For each of the three groups of Type II subjects in the present experiments (100-msec condition in Experiment $1,3,000-$ msec condition in Experiment 1, and

Figure 6. Mean reaction time as a function of probability of response. Both correct and incorrect reaction times are plotted. The data of both Type I and Type II subjects for the 0-msecinterstimulus-interval condition are shown in the upper two panels. The data of both Type I and Type II subjects for the 3,000 -msec-interstimulus-interval condition are shown in the lower two panels.

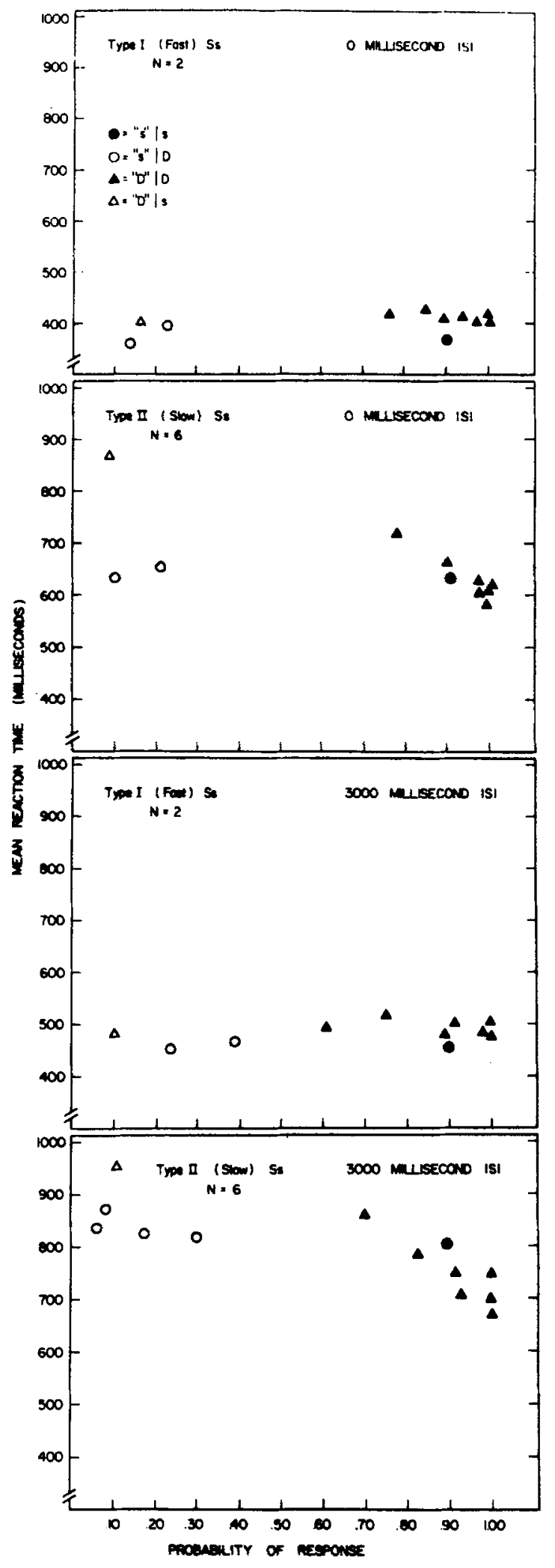


the average of both conditions in Experiment II), these rank-order correlations were above +.90 , and for all, $p<.01$. For the Type I subjects, whose "different" reaction times were not affected by similarity between the standard shape and the test form, all correlations were extremely small and nonsignificant.

Two invitingly simple explanations for the Type IType II performance differences can be discarded on the basis of the constellation of results from Experiments I and II. First, the possibility that a "floor effect" might explain the flat "different" reactiontime functions for the Type 1 subjects can be rejected because the "same" responses of these subjects are even faster than their "different" responses (cf. Figures 3 through 6). Second, the possibility that a speed-accuracy tradeoff might somehow explain the subject differences is unlikely for the following reasons: (a) Neither type of subject consistently made more errors than the other type of subject (cf. Tables 1 and 2). (b) Neither type of subject consistently made more errors to different than to same test probes (or vice versa, cf. Tables 1 and 2). (c) Despite the marked differences in the pattern of the reaction-time data for the two types of subjects, the pattern of their error rates associated with each class of test probe are quite comparable (cf. Figures 3 through 6 and Tables 1 and 2).

The Type I-Type II performance differences have implications for models of the same-different visual comparison process, particularly in light of the inadequacy of the simple explanations discussed above. Consider, first, the performance of the Type I subjects. Neither the single-process nor the dualprocess models outlined in the introduction can explain the Type I data, because both classes of models postulate a self-terminating feature comparison which should produce longer "different" responses as the stimuli being compared become more similar.

A more plausible model for the performance of Type I subjects postulates a rapid, holistic comparison of a visual memory representation with a visually presented test form. If the outcome of this holistic comparison produces a match, then the "same" response is executed. If the comparison fails to produce a match, then extra time is required to execute the "different" response. Under this account, the "different" response is made by default (only when the comparison process cannot verify that the memory representation and the test stimulus are the same). Thus, the "different" response should be executed with uniform speed regardless of the similarity between the test probe and the standard shape. Both the correct and the incorrect reaction-time data of Type I subjects are consistent with this simple single-process model. This is because all "same" responses and all "different" responses are made equally rapidly, regardless of whether or not they are accurate (cf. Figures 4 and 6 ).

The pattern of errors made by Type I subjects (cf. Tables 1 and 2) is also consistent with a model which postulates a single, holistic comparison. This is because the memory representation of the standard shape is probably a somewhat degraded or incomplete representation of the standard. Thus, the majority of errors will occur when the comparison process fails to detect a match (owing to the partially degraded memory representation of the standard), and also when the memory representation contains insufficient information to permit rejection of a different probe which is highly similar to the standard. In addition, the higher error rates obtained with long interstimulus intervals (cf. Table 2) can be explained by this simple model. That is, the somewhat incomplete memory representation of the standard shape presumably becomes even more degraded with time, thereby resulting in more errors of the sort outlined above.

Note, however, that one feature of the data of Type I subjects from Experiment II cannot be readily explained-viz, the longer reaction times obtained with the longer interstimulus interval (cf. Figures 5 and 6). Presumably, the holistic, parallel nature of the comparison process results in nearly uniform "different" response speeds, regardless of the discriminability of the standard and the test probe. Why, then, should further degradation of the memory representation of the standard, resulting from the long interstimulus interval, produce longer comparison times when this degradation serves only to reduce further the discriminability of the memory representation and the test probe?

The performance of the Type 11 subjects is not consistent with the class of single-process models which assume a self-terminating comparison of the features of two visual representations. This is because the "same" response, while slower than the average "different" response, is still faster than "different" responses to test probes which are highly similar to the standard (cf. Figures 3 through 6). The data of the Type II subjects are, however, easily explained by dual-process models which assume independence of the processes underlying the "same" and the "different" responses. The intermediate speed of the "same" response suggests that the two processes operate simultaneously. The monotonic decrease in "different" reaction time with increasing dissimilarity between the standard shape and the test probe suggests that the "different" processor compares features of the two visual representations, checking for a difference in shape. The greater the dissimilarity between the memory representation of the 
standard and the visual test probe, the earlier a difference will be found and, thus, the faster will be the "different" response. The "same" processor is assumed to operate in a manner similar to the single holistic comparison process postulated for Type I subjects. That is, the processor attempts to verify that the memory representation of the standard and the visual test probe are the same, and if a match is found the "same" response is executed. The crucial difference between the holistic Type I comparison process and the processor underlying the "same" responses of Type II subjects is that the latter process cannot give any output concerning a mismatch, i.e., a "different" response (cf. Bamber, 1969).

The incorrect reaction-time data of Type II subjects shown in Figures 4 and 6 provide strong support for a dual-process model. Note that for all three groups of Type II subjects (100-msec condition in Experiment $1,3,000$-msec condition in Experiment I, and both conditions in Experiment II), both incorrect and correct "same" responses are approximately equal in speed. This is precisely what we should expect if the "same" processor operates independently and holistically, providing output only when sufficient evidence for a match has been obtained (whether or not this evidence is accurate or inaccurate). In addition, note that incorrect "different" responses are considerably longer than all correct "different" responses. Again, this is precisely what we should expect if the "different" processor operates independently and performs a self-terminating comparison of the features of the memory representation of the standard and the features of the test probe. When the test probe is, in fact, the same as the standard, it takes considerable time for the "different" processor to obtain (inaccurate) evidence for a mismatch and output the "different" response. Unfortunately, two-process models are obviously uneconomical in that only one process is logically sufficient to make the same-different comparison. (This is clear from the above analysis of the processes underlying Type I performance.) Nonetheless, the sort of dual-process model outlined above provides an excellent account of the performance of Type II subjects.

Recently, Hock and his associates (Hock, 1973; Hock, Gordon, \& Marcus, 1974) have reported individual differences in same-different visual comparison which may be related to the Type I-Type II differences reported here. Specifically, Hock has found that certain stimulus manipulations such as symmetry, familiarity (varied by rotating the stimulus from a previously learned orientation), and embeddedness affect the "same" response speed of some subjects and not of others. "Different" reaction times do not appear to be affected by these stimulus manipulations. Hock describes the subjects whose "same" responses are facilitated by these manipulations as "structural" subjects. Subjects whose "same" responses are unaffected are termed "analytic' subjects.

The terms which Hock uses to describe these performance differences are quite descriptive of the processing differences hypothesized to underlie Type I and Type II performance. However, it is not clear whether there is any relationship between Hock's results and the results of the present experiments. There are significant methodological differences between the Hock studies and the present experiments. In addition, Hock's differences are apparent only in "same" reaction times, while the Type I-Type II differences are found in the pattern of both "same" and "different" responses. Finally, Hock's "structural" subjects-whose processing would presumably be similar to that of Type I subjects-have longer reaction times than the "analytic" subjects-whose processing would presumably be similar to that of Type II subjects. The situation is reversed in the case of the subjects in the present experiment, for Type I subjects have unusually rapid response times. Hopefully, further research can clarify the possible relationship between the "structural"'-"analytic" performance difference and the Type I-Type II performance differences.

In summary, the individual differences in samedifferent visual comparison reported by Cooper and Podgorny (in press) have been found again in the present experiments. Of the 24 subjects studied thus far (6 in the Cooper-Podgorny experiment and 18 in the present experiments), 17 have shown Type II performance while only 7 have shown Type 1 performance. If these samples are at all representative of the population of subjects used in most visual comparison experiments, then the relative paucity of Type I subjects may explain why this pattern of performance has not been detected by other investigators who generally report only group data. Further research concerning the nature of the processing differences which underlie Type I and Type II performance is currently in progress. Questions being explored include how obligatory these comparison strategies are and whether these comparison strategies generalize to a variety of stimulus and task domains. Hopefully, such research will clarify the nature of the Type I-Type II processing difference and lead to a more detailed model of same-different visual comparison.

\section{REFERENCES}

Attneave. F. Physical determinants of the judged complexity of shapes. Joumal of Experimental Psychology. 1957. 53. $221-227$

Attneave. F.. \& Arnoult. M. D. The quantitative study of shape and pattern perception. Psychological Bulletin. 1956. 53. $452-471$. 
BAMBER. D. Reaction times and error rates for "same".."different" judgments of multidimensional stimuli. Perception \& Psychophysics. 1969. 6. 169-174.

BAMBER. D.. \& PAINE. S. Information retrieval processes in "same"." different" judgments of letter strings. In S. Kornblum (Ed.). Attention and Pertormance IV. New York: Academic Press. 1973.

Bindra. D., Donderi. D. C.. \& Nishisato, S, Decision latencies of "same" and "different" judgments. Perception \& Psychophysics. 1968, 3, 121-130.

Cooper. L. A. Mental rotation of random two-dimensional shapes. Cognitive Psychology, 1975, 7, 20-43.

Cooper. L. A.. \& Podgorny, P. Mental transformations and visual comparison processes: Effects of complexity and similarity. Journal of Experimental Psychology: Human Percep. tion and Pertormance. in press.

Cooper. L. A.. \& Shepard. R. N. Chronometric studies of the rotation of mental images. In W. G. Chase (Ed.). Visual informution processing. New York: Academic Press. 1973.

EgEth. H. Parallel versus serial processes in multidimensional stimulus discrimination. Perception \& Psychophysics. 1966. 1. $245-252$.

Egeth, H. \& Blecker. D. Differential effects of familiarity on judgments of sameness and difference. Perception \& Psychophisics. 1971. 9. 321.326.

Hawkins. H. L. Parallel processing in complex visual discrimination. Perception \& Psychophysics. 1969. 5. 56:64.

Hock. H. S. The effects of stimulus structure and familiarity on same-different comparison. Perception \& Psychophysics. 197.3. 14. 413-420.

Hock. H. S.. Gordon. G. P.. \& Marcus, N. Individual differences in the detection of embedded figures. Perception \& Psychophysics. 1974. 15. 47.52.

KRUEGER. L. E. Effect of bracketing lines on speed of "same". "different" judgment of two adjacent letters. Journal of Experimental Psichologi. 1970. 84. 324-330.

KRUEGER. L. E. Effect of stimulus frequency on speed of "same""ditferent" judgments. In S. Kornblum (Ed.). Attention and performance IV. New York: Academic Press. 1973.
Nickerson. R. S. "Same"-"different" response times with multi-attribute stimulus differences. Perceptual and Motor Skills. 1967, 24, 543-554.

Nickerson. R. S.. "Same".-"different" response times: A model and a preliminary test. Actu Psychologica, 1969. 30. 257-275.

Nickerson. R. S. Binary-classitication reaction time: A review of some studies of human information-processing capabilities. Psychonomic Monograph Supplements, 1972. 4. (Whole N(i. 65), 275-318.

Sekuler. R. W.. d Abrams. M. Visual sameness: A choice time analysis of pattern recognition processes. Joumal of Exprerimo'mul Psychology. 1968. 77, 232.238.

Shepard. R. N.. \& Metzler. J. Mental rotation of threedimensional otjects. Scionce, 1971, 171, 701-703.

TVERSKY. B. Piclorial and verbal encoding in a short term memory lask. Porieption \& Psychophesics. 1969. 6. 225-233.

\section{NOTES}

1. Throughout, the identity of responses and reaction times will be placed in quotations. The nature of trial types and stimulus pairs will not be placed in quotations. For example, the expression, probability ("same" | same), refers to the probability of a "same" response given that the pair of stimuli were, in fact, the same.

2. Following the experiment, the same 10 subjects were called back in order to determine whether the time to say " $S$ " differed from the time to say "D." For each of the 10 subjects, 40 trials were run. On 20 of the trials, the letter $S$ was presented and on 20 of the trials, the letter D was presented. Subjects were required to name the presented letter as rapidly as possible. Mean time to say " $S$ " was $430 \mathrm{msec}$, and mean time to say " $D$ " was $423 \mathrm{msec}$. For individual subjects, the difference between " $S$ " and " $D$ " response times ranged from 30 to $2 \mathrm{msec}$.

(Received for publication November 19, 1975; accepted February 17, 1976.) 